\title{
How faculae and network relate to sunspots, and the implications for solar and stellar brightness variations (Corrigendum)
}

\author{
K. L. Yeo ${ }^{1}$, S. K. Solanki ${ }^{1,2}$, and N. A. Krivova ${ }^{1}$ \\ ${ }^{1}$ Max-Planck Institut für Sonnensystemforschung, Justus-von-Liebig-Weg 3, 37077 Göttingen, Germany \\ e-mail: yeo@mps.mpg.de \\ 2 School of Space Research, Kyung Hee University, Yongin, 446-701 Gyeonggi, Korea \\ A\&A 639, A139 (2020), https: //doi .org/10.1051/0004-6361/202037739
}

Key words. Sun: activity - Sun: faculae, plages - Sun: magnetic fields - sunspots - errata, addenda

This is a corrigendum to Yeo et al. (2020). We had listed the wrong values for the parameter $h_{1}$ in Table 1 . The error is purely typographical and confined to this table, and as such there is no impact on the rest of the paper. The revised table is given below.

\section{References}

Yeo, K. L., Solanki, S. K., \& Krivova, N. A. 2020, A\&A, 639, A139

Table 1. Similar to Table 1 in Yeo et al. (2020), except with the correct values of $h_{1}$.

\begin{tabular}{lccccc}
\hline \hline$F$ & $S$ & $h_{1}$ & $h_{2}$ & $h_{3}$ & $h_{4}$ \\
\hline$F_{10.7}$ & $S_{\mathrm{A}}$ & $1.369 \times 10^{-2}$ & 0.8603 & 24.35 & 64.38 \\
$F_{\mathrm{CaIIK}}$ & $S_{\mathrm{A}}$ & $5.030 \times 10^{-6}$ & 0.5854 & 57.41 & $8.228 \times 10^{-2}$ \\
$F_{L \alpha}$ & $S_{\mathrm{A}}$ & $7.198 \times 10^{-7}$ & 0.6642 & 72.34 & $5.852 \times 10^{-3}$ \\
$F_{\mathrm{MgII}}$ & $S_{\mathrm{A}}$ & $3.256 \times 10^{-6}$ & 0.7129 & 54.10 & 0.1497 \\
$F_{\phi}$ & $S_{\mathrm{A}}$ & $1.992 \times 10^{-4}$ & 0.8429 & 33.46 & 0.1824 \\
\hline$F_{10.7}$ & $S_{\mathrm{N} 1}$ & $7.334 \times 10^{-2}$ & 1.073 & 13.41 & 65.10 \\
$F_{\mathrm{C} \text { CIIK }}$ & $S_{\mathrm{N} 1}$ & $1.357 \times 10^{-5}$ & 0.7913 & 34.84 & $8.243 \times 10^{-2}$ \\
$F_{L \alpha}$ & $S_{\mathrm{N} 1}$ & $3.230 \times 10^{-6}$ & 0.7960 & 49.25 & $5.861 \times 10^{-3}$ \\
$F_{\mathrm{MgII}}$ & $S_{\mathrm{N} 1}$ & $1.614 \times 10^{-5}$ & 0.8648 & 33.25 & 0.1498 \\
$F_{\phi}$ & $S_{\mathrm{N} 1}$ & $1.735 \times 10^{-3}$ & 0.9572 & 20.15 & 0.1401 \\
\hline$F_{10.7}$ & $S_{\mathrm{N} 2}$ & $4.573 \times 10^{-2}$ & 1.092 & 13.42 & 65.44 \\
$F_{\mathrm{CaIIK}}$ & $S_{\mathrm{N} 2}$ & $1.078 \times 10^{-5}$ & 0.7846 & 34.52 & $8.254 \times 10^{-2}$ \\
$F_{L \alpha}$ & $S_{\mathrm{N} 2}$ & $2.082 \times 10^{-6}$ & 0.8346 & 46.47 & $5.898 \times 10^{-3}$ \\
$F_{\mathrm{MgII}}$ & $S_{\mathrm{N} 2}$ & $8.884 \times 10^{-6}$ & 0.9209 & 32.71 & 0.1501 \\
$F_{\phi}$ & $S_{\mathrm{N} 2}$ & $1.054 \times 10^{-3}$ & 0.9841 & 20.26 & 0.1401 \\
\hline$F_{10.7}$ & $S_{\mathrm{G} 1}$ & 0.7818 & 1.126 & 19.53 & 64.01 \\
$F_{\mathrm{CaIIK}}$ & $S_{\mathrm{G} 1}$ & $5.693 \times 10^{-5}$ & 0.9559 & 35.56 & $8.336 \times 10^{-2}$ \\
$F_{L \alpha}$ & $S_{\mathrm{G} 1}$ & $1.501 \times 10^{-5}$ & 0.9295 & 54.62 & $5.887 \times 10^{-3}$ \\
$F_{\mathrm{MgII}}$ & $S_{\mathrm{G} 1}$ & $1.156 \times 10^{-4}$ & 0.9539 & 31.37 & 0.1495 \\
$F_{\phi}$ & $S_{\mathrm{G} 1}$ & $1.590 \times 10^{-2}$ & 0.9538 & 23.28 & 0.1898 \\
\hline$F_{10.7}$ & $S_{\mathrm{G} 2}$ & 0.6196 & 1.255 & 19.68 & 63.91 \\
$F_{\mathrm{CaIIK}}$ & $S_{\mathrm{G} 2}$ & $7.421 \times 10^{-5}$ & 0.9240 & 36.82 & $8.218 \times 10^{-2}$ \\
$F_{L \alpha}$ & $S_{\mathrm{G} 2}$ & $1.564 \times 10^{-5}$ & 1.002 & 48.40 & $5.850 \times 10^{-3}$ \\
$F_{\mathrm{MgII}}$ & $S_{\mathrm{G} 2}$ & $8.279 \times 10^{-5}$ & 1.105 & 34.04 & 0.1499 \\
$F_{\phi}$ & $S_{\mathrm{G} 2}$ & $1.361 \times 10^{-2}$ & 1.072 & 23.83 & 0.1279 \\
\hline & & & & &
\end{tabular}

